\title{
REFLEXIONES EN TORNO AL LENGUAJE MÉDICO ACTUAL, LOS EPÓNIMOS Y ABREVIACIONES. LAS RAZONES DE SU EXISTENCIA Y LOS PRINCIPALES PROBLEMAS QUE PLANTEA SU USO
}

Juan Carlos Araujo C. ${ }^{1}$

\section{RESUMEN}

El lenguaje médico, como todo lenguaje científico, no persigue fines estéticos, creativos, lúdicos ni recreativos como sería el caso del lenguaje literario, sino fines informativos, didácticos y comunicativos. Los epónimos, así como los diferentes tipos de abreviaciones (abreviaturas, siglas y símbolos), han formado parte del lenguaje de la humanidad desde más de una veintena de siglos y son especialmente abundantes en los lenguajes de las especialidades, tanto de la medicina como de otras ciencias. Así como del lenguaje común y popular, estos han formado parte del lenguaje desde tiempos inmemoriales, y son especialmente abundantes en los lenguajes médico-científicos. Normalmente se utilizan como recurso para honrar, ganar tiempo y ahorrar espacio, cuando esté en su contenido se percibe bien, su uso revela notoriamente un ahorro en la comunicación médica; de no emplearlos, en cada caso habría que hacer una exposición o descripción relativamente amplia de la enfermedad o el signo de que se trata. Sin embargo, pueden producir problemas de comunicación entre los profesionales de los diferentes niveles asistenciales, debido a su imprecisión y a su carencia de equivalencia internacional, lo que puede llevar a confundir la realidad que tratan de describir, ya que un mismo término o abreviatura puede tener diferentes significados, lo que puede suscitar interpretaciones erróneas con posibles consecuencias graves. En este artículo se revisan las razones de su existencia que definen su construcción y uso, se describen los problemas de su utilización en la comunicación medico científica en la actualidad.

Palabras clave: epónimo, abreviaciones, abreviaturas, siglas, acrónimos, lenguaje médico, razón, existencia, problema.

\section{REFLECTIONS ON CURRENT MEDICAL LANGUAGE. THE EPONYMS AND ABBREVIATIONS. THE REASONS OF ITS EXISTENCE AND THE MAIN PROBLEMS THAT RAISE ITS USE}

\section{ABSTRACT}

Medical language, like all scientific language, does not pursue aesthetic, creative, leisure and recreational purposes as would be the case in literary language, but informative, educational and communication purposes. Eponyms and the different types of abbreviations (abbreviations, acronyms, and symbols) have been part of the language of humanity for over twenty centuries and are especially abundant in the

\footnotetext{
${ }_{1}$ Profesor Titular. Facultad de Medicina. Escuela de Medicina. Universidad del Zulia (LUZ), Especialista en cirugía general. Correo electrónico: jcaraujoc_65@hotmail.com; jcaraujoc95@gmail.com.
} 
languages for both specialties in medicine, and other sciences. On the common and popular language, these elements have been part of the language since ancient times, and are especially abundant in the medical-scientific languages. Normally are used as a resource to honor, to save time and save space, when its content is perceived well, notoriously it reveals a saving in medical communication; not to use them, in each case would have to make a relatively large exposure or description of the disease or of the sign in question. However, they can cause communication problems between professionals of different levels of care, due to their imprecision

\section{INTRODUCCIÓN}

Como bien es conocido, el lenguaje permite al ser humano fragmentar la masa deforme e indivisa que es la realidad, estableciendo en ella distinciones y categorías que se convierten en el contenido de las palabras de cada lengua concreta. El uso apropiado y preciso de un lenguaje específico sobre un área de conocimiento es crucial para la buena comunicación. En el mundo de la medicina, y lo que refiere a ella, se suelen utilizar términos médicos, con epónimos, abreviaturas, sigla y símbolos, lo que llamamos el lenguaje médico. Los términos del lenguaje médico se usan para intercalar precisión, neutralidad y facilitar las expresiones que día a día se utilizan en las distintas áreas de la medicina $(1,2)$.

Su comprensión resulta ser muy relevante sobre todo para aquellos profesionales, que trabajan o piensan trabajar (estudiantes) en el cuidado de la salud y, emplean cotidianamente términos médicos más familiares como: sida, enfermedad de Parkinson, síndrome de Down, carcinoma (Ca), entre otros; en muchas ocasiones desconociendo el valor histórico de los mismos. Resultaba muy habitual denominar con un nombre epónimo enfermedades, síndromes, and their lack of international equivalence, which may lead to confusing the reality they are trying to describe since the same term or abbreviation could have different meanings, which can lead to misinterpretations with potentially serious consequences. This article discusses the reasons for their existence which define their construction and uses, problems of its use in medical scientific communication at present are also described.

Key words: eponymous, abbreviations, acronyms, medical language, reason, existence problems.

signos, instrumentos, partes anatómicas y pruebas clínicas con los nombres de sus descubridores o inventores e inclusive abreviarlo incluyendo con el uso de siglas o símbolos. Son ampliamente utilizados en todos los campos científicos y fundamentalmente en las ciencias médicas, utilizándose habitualmente como un recurso para ganar tiempo y ahorrar espacio $(3,4)$.

El lenguaje médico, como ya hemos indicado, es uno de los lenguajes científicos con mayor proliferación en el uso de nombres epónimos y abreviaturas. Los epónimos han formado parte del lenguaje desde tiempos ancestrales, y son especialmente abundantes en los lenguajes de los profesionales dedicados al cuidado de la salud. Pero definamos que es un epónimo, este proviene del vocablo griego $\varepsilon \pi t-($ epi $=$ encima, sobre) y $\omega v v \mu$ os (onomos = nombre). Por lo cual el diccionario de la Real Academia Lengua Española, define a un epónimo como “el nombre de una persona o de un lugar que designa un pueblo, una época, una enfermedad, una unidad, entre otros (5). Entonces, podemos decir que nos encontramos ante un fenómeno muy extendido y que ha existido durante gran parte de la historia de la humanidad y por lo tanto de la medicina. 
Los epónimos no solo se limitan a los nombres, sino que también algunos epónimos se derivan de aspectos históricos, geográficos, literarios y hasta inclusive mitológicos. En la actualidad, en lo que refiere a medicina existen cerca de 9.000 epónimos, siendo los más significativos en su respectiva área, como por ejemplo, Acueducto de Silvio, que se refiere al conducto que comunica el tercero con el cuarto ventrículo del cerebro bautizado así en honor al médico, anatomista y fisiólogo francés Franciscus Sylvius (6).

Sin embargo, la eponimia no tiene un uso reciente, sino que su uso se ha generalizado, se ha extendido y ha coexistido durante gran parte de la historia del mundo científico, algunos de los más antiguos datan del primer y segundo milenio antes de Cristo $(5,6)$.

Por otro lado, la abreviatura es la representación de una palabra o de las palabras de una frase por alguna o algunas de sus letras, la primera de las cuales ha de ser la inicial de la palabra abreviada. Las abreviaturas conservan el género y el número de la palabra completos, se pueden formar siguiendo unas normas llamadas a estas regulares, pudiendo ser de suspensión o de contracción y si no siguen ninguna sistemática son llamadas irregulares. Las abreviaturas son recursos para ahorrar tiempo y espacio en el lenguaje, pero su proliferación genera dificultades de comprensión debido a que son instrumentos imprecisos y peligrosos, sujetos al capricho del creador, lo que puede conducir a confundir la realidad que tratan de describir, ya que una misma abreviación puede tener diferentes significados (7).

El Diccionario de la Lengua Española menciona las abreviaturas, siglas y acrónimos, y los problemas que conlleva el uso y abuso de estas abreviaciones, producto muchas veces de su imprecisión, su falta de normalización, y el hecho de que pueden tener varios significados. Presenta, también, las principales reglas que las rigen: no se puntúan, no se separan por espacios, no tienen plural, deben descifrarse la primera vez que se utilizan en un texto, pueden sustantivarse y su género es el de la primera palabra que la componen.

En el lenguaje científico, el abuso de epónimos, abreviaturas y siglas, que no han sido internacionalmente aceptados por los comités de normalización, lo convierten en un instrumento impreciso, con graves problemas para su comprensión y pueden conllevar a interpretaciones erróneas que pueden producir graves consecuencias. En ocasiones se establecen por simple economía lingüística del creador. Además, evolucionan, aparecen otras nuevas que las sustituyen, pierden o cambian de significado, se utilizan en nuevas situaciones en las que es difícil reconocerlas o caen en desuso por el abuso de acrónimos que pueden volver ininteligible un texto $(5,6,7)$.

Son abundantes las dificultades que tienen los diversos profesionales de la salud que intervienen en la atención medica al momento de poder entender o interpretar adecuadamente la información suministrada por el colega sobre los pacientes y la literatura publicada, la cual se debe en numerosas ocasiones a la presencia en el lenguaje médico cotidiano de abreviaciones y otras siglas en los diversos documentos relacionados con la asistencia y la investigación médico-científica, estas son abundantes en los documentos como la historia clínica, la hoja de referencia o interconsulta entre especialidades, los informes de alta y otros tipos de informes, así como en los artículos publicados en revistas e inclusive en las monografías científicas $(7,8)$.

Durante las últimas décadas, la devaluación científica, social y económica de los profesionales de la salud, ha producido un avanzado e inquietante deterioro del lenguaje médico. Se señalan las numerosas situaciones con que se topan los médicos; problemas de diagnósticos médicos que se reflejan en las historias clínicas de sus colegas, debido al uso exagerado de epónimos, abreviaciones o siglas para referirse a un determinado termino médico. 
La utilización de estos recursos lingüísticos de forma cotidiana, puede hacer parecer que se tiene un elevado conocimiento de la materia en cuestión; sin embargo, está demostrado que su uso en demasía puede hacer ininteligible la comunicación, y confundir a otros profesionales que no están identificados con ellas, es decir su uso abusivo produce más dificultades de comprensión.

Por una parte, es muy lastimoso que, en el lenguaje médico, muchas veces se prefiera a la "sopa de letras" originadas por el uso abusivo de los nombres epónimos y de las abreviaciones a lo atractivo y la finura de la terminología española de raíz grecolatina. Debido a la escasa formación actual de los participantes del pregrado e inclusive los de posgrado, quienes han transformado la toma de apuntes en régimen de economato, y la desvalorización de las lenguas clásicas en las instituciones educativas como las facultades de medicina, los colegios de médicos, las academias y las sociedades científicas, que deben ser vigilantes de la regulación en la utilización de nombres epónimos, abreviaturas y siglas en los tests o pruebas de evaluación, informes médicos y artículos científicos, entre otros (8).

Y por otra parte, el problema en el crecimiento de la terminología médica, con la incorporación constante de neologismos y de la falta de normalización se añade el de la traducción o adaptación de los términos que se acuñan en inglés. Si este proceso, que es inevitable, se sigue realizando de forma anárquica, se corre el riesgo de que el español resulte cada vez un lenguaje más incómodo para la comunicación científica, agravado por la proliferación de abreviaciones y el descuido de los aspectos sintácticos $(7,8)$.

\section{La eponimia y las abreviaciones en el lenguaje médico}

Cualquier vocabulario es un sistema abierto, y a esta característica no podía escapar el lenguaje médico, debido a que como sistema en eterna reconcepción por su condición de inestabilidad, inherente a la evolución de todo conocimiento científico. Está formado por palabras o expresiones de orígenes diversos, referencias geográficas, literarias y mitológicas, nombres propios de personas célebres, abreviaciones, metáforas, neologismos, barbarismos, acrónimos, símbolos y siglas. Es por ello que uno de los aspectos más característicos del lenguaje en la medicina, es el uso habitual de epónimos o términos construidos sobre nombres propios. Los epónimos bien conocidos por la comunidad científica son expresiones que incorporan los apellidos de científicos para denominar ciclos, enfermedades, leyes, pruebas, reacciones, signos o síndromes. Asimismo, los epónimos no se aplican a enfermedades y síndromes, sino que igualmente se designan con ellos a signos, síntomas, tratamientos, intervenciones quirúrgicas, maniobras diagnósticas, reacciones fisiológicas, posiciones, instrumental médico, términos anatómicos, reactivos, análisis, microorganismos y anticuerpos (10).

Sin embargo, las definiciones anteriores son incompletas si se aplican a nuestro ámbito. En medicina, los epónimos tienen, por extensión, un espectro de acción mucho más amplio que la sola honra de nuestros héroes o lugares, también inmortalizan a personajes médicos, apellidos de pacientes, lugares geográficos, instituciones, figuras bíblicas y seres mitológicos $(2,4)$. Los epónimos son miembros de pleno derecho del conjunto de términos de los que dispone un dominio científico o técnico para designar objetos y conceptos propios y, por ello, se comportan como los demás signos lingüísticos, con sus problemas de sinonimia, homonimia y polisemia $(10,11)$. Como complemento a lo expresado, se confeccionó una lista de los epónimos más comunes en el lenguaje médico, como se expone en la tabla 1 .

En la escritura siempre llega el momento en que se necesita acortar, reducir o hacer breves las palabras y expresiones para decir mucho con poco. Las abreviaciones son unas 
representaciones abreviadas de una o varias palabras. Las abreviaciones y sus diferentes tipos (abreviaturas, siglas y símbolos) son ampliamente empleadas en todos los campos científicos y especialmente en el campo medico, utilizándose normalmente como un recurso para ganar tiempo y ahorrar espacio (7-9).

Tabla 1. Epónimos más comunes en el lenguaje médico.

\begin{tabular}{|c|c|}
\hline NOMBRE DEL AUTOR & EPÓNIMO \\
\hline Addison, Thomas (1793-186 & ENFERMEDAD, ANEMIA, QUELOIDE DE ADDISON \\
\hline Alibert, Jean Louis (1768-1837) & ENFERMEDAD, QUELOIDE DE ALIBERT \\
\hline Argumosa Obregón, Diego (1792-1865) & ARGUMOSA OBREGÓN, DIEGO \\
\hline Aschoff, Karl Albert Ludwig (1866-1942) & NUDO O NODO, CUERPO DE ASCHOFF \\
\hline Babinski, Joseph François (1852-1932) & REFLEJO, SIGNO, SÍNDROME DE BABINSKI \\
\hline Banting, Frederick Grant (1891-1941) & TRATAMIENTO DE BANTING \\
\hline Bárány, Robert (1876-1936) & SINGO, SÍNDROME DE BÁRÁNY \\
\hline Barraquer I, Barraquer, Ignasi (1884-1965) & OPERACIÓN, TÉCNICA, PROCEDIMIENTO DE BARRAQUER \\
\hline Barraquer I, Roviralta, Lluis (1855-1928) & SÍNDROME DE BARRAQUER, REFLEJO DE BARRAQUER \\
\hline Barré, Jean Alexandre (1880-1967) & SÍNDROME DE GUILLAIN-BARRÉ, SIGNO, PRUEBA DE BARRÉ \\
\hline Bartholin, Caspar (1585-1629) & BARTHOLIN, CASPAR \\
\hline Behring, Emil Adolf von (1854-1917) & SUERO DE BEHRING \\
\hline Bell, Charles (1774-1842) & ENFERMEDAD, PARÁLISIS, NERVIO, LEY, FENÓMENO DE BELL \\
\hline Bichat, François Xavier (1771-1802) & CISURA, LIGAMENTO, TEJIDO DE BICHAT \\
\hline Bilharz, Theodor Maximilian (1825-1862) & BILHARZIA, BILHARZIASIS \\
\hline Billroth, Theodor (1829-1894) & OPERACIÓN DE BILLROTH \\
\hline Boerhaave, Hermann (1668-1738) & SÍNDROME DE BOERHAAVE \\
\hline Bordet, Jules (1870-1961) & BORDETELLA PERTUSSIS \\
\hline Bouchard, Charles Jacques (1837-1915) & $\begin{array}{l}\text { ANEURISMAS DE CHARCOT-BOUCHARD, SIGNO DE } \\
\text { BOUCHARD }\end{array}$ \\
\hline Bright, Richard (1789-1859) & ENFERMEDAD DE BRIGHT \\
\hline Brissaud, Édouard (1852-1909) & ENFERMEDAD DE BRISSAUD \\
\hline Brown-Séquard, Charles Edouard (1817-1894) & SÍNDROME, PARÁLISIS... DE BROWN-SÉQUARD \\
\hline Burkitt, Denis Parsons (1911-1993) & LINFOMA DE BURKITT \\
\hline Calleja Sánchez, Julián (1836-1913) & ISLOTE DE CALLEJA \\
\hline Calmette, Albert (1863-1933) & VACUNA DE BACILO CALMETTE-GUERIN \\
\hline Cannon, Walter Bradford (1872-1945) & ANILLO DE CANNON \\
\hline Carrel, Alexis & MÉTODO, TRATAMIENTO DE CARREL \\
\hline
\end{tabular}


Juan Carlos Araujo C.

\begin{tabular}{|c|c|}
\hline NOMBRE DEL AUTOR & EPÓNIMO \\
\hline Charcot, Jean Martin (1825-1893) & TRÍADA DE CHARCOT \\
\hline Cheyne, John (1777-1836) & RESPIRACIÓN DE CHEYNE-STOKES \\
\hline Cloquet, Jules Germain (1790-1883) & GANGLIO, HERNIA, CONDUCTO DE CLOQUET \\
\hline Colles, Abraham (1773-1843) & FASCIA, LIGAMENTO, FRACTURA, LEY DE COLLES \\
\hline Cooper, Astley Paston (1768-1941) & APONEUROSIS, LIGAMENTO, ENFERMEDAD DE COOPER \\
\hline Corvisart, Jean-Nicolas & FACIES, ENFERMEDAD \\
\hline Councilman, William Thomas (1854-1933) & CUERPOS DE COUNCILMAN \\
\hline Courvoisier, Ludwig (1843-1913) & SÍNDROME DE COURVOISIER-TERRIER \\
\hline Creutzfeldt, Hans-Gerhard (1885-1964) & ENFERMEDAD DE CREUTZFELDT-JAKOB \\
\hline Cruveilhier, Jean (1791-1874) & $\begin{array}{l}\text { ATROFIA DE CRUVEILHIER, SÍNDROME DE CRUVEILHIER- } \\
\text { PEAN }\end{array}$ \\
\hline Curling, Thomas Blizard (1811-1888) & ÚLCERA DE CURLING \\
\hline Cushing, Harvey Williams (1869-1939) & SÍNDROME DE CUSHING \\
\hline Dejerine, Joseph Jules (1849-1917) & SÍNDROME, SIGNO, ENFERMEDAD DE DEJERINE \\
\hline Dieulafoy, Georges Paul (1839-1911) & APARATO, ENFERMEDAD, TRÍADA, TEORÍA DE DIULAFOY \\
\hline Dolbeau, Henri Ferdinand (1830-1877) & LITOTRICIA PERINEAL DE DOLBEAU \\
\hline Donders, Franz Cornelius (1818-1889) & GLAUCOMA, ENFERMEDAD, LEY DE DONDERS \\
\hline Doppler, Christian Johann (1803-1853) & EFECTO DOPPLER \\
\hline Down, John Langdon (1828-1896) & SÍNDROME DE DOWN \\
\hline Hodgkin, Thomas (1798-1866) & LINFOMA DE HODKING \\
\hline Duchenne, Guillaume B. A. (1806-1875) & ENFERMEDAD DE DUCHENNE \\
\hline Fanconi, Guido (1892-1979) & ENFERMEDAD DE DUCHENNE AMIA DE FANCONI \\
\hline Ménière, Prosper (1799-1862) & SÍNDROME DE MÉNIÈRE \\
\hline Morgagni, Giovanni Battista (1682-1771) & HERNIA DE MORGAGNI \\
\hline Ricketts, Howard Taylor (1871-1910) & RICKETTSIAS \\
\hline Salmon, Daniel Elmer (1850-1914) & SALMONELLA \\
\hline Schwann, Friedrich Theodor (1810-1882) & CÉLULAS DE SCHWANN \\
\hline Shiga, Kiyoshi (1871-1957) & SHIGELLA, SHIGELOSIS \\
\hline
\end{tabular}

Una abreviatura (del latín abreviatura, de abreviare, "hacer breve") es un tipo de abreviación, una convención ortográfica que acorta la escritura de cierto término o expresión, y consiste en la representación escrita de una palabra o grupo de palabras con solo una o varias de sus letras, la primera de las cuales ha de ser la inicial de la palabra abreviada, en mayúscula o minúscula, por sí sola o acompañada de otras letras, ya sean del medio o del fin de dicha palabra, y uno o varios puntos para indicar que la palabra está incompleta. Es decir, entonces de ella que está 
escrita abreviadamente. Por ejemplo, “a.C.: es la abreviatura de antes de Cristo" (8).

Las abreviaturas fueron muy empleadas en Grecia y en Roma; se abusó tanto de ellas, que el emperador Justiniano se vio obligado a prohibir su empleo. En Francia, ante abuso semejante, el rey Felipe el Hermoso prohibió, en 1304, que se emplearan en los documentos notariales y en general, en los instrumentos públicos aquellas abreviaturas que pudieran dar lugar a confusión o mala interpretación de los textos. Así tenemos entonces varios ejemplos en el lenguaje medico como: electro (electrocardiograma o electroencefalograma), gine (ginecología o ginecólogo) y quimio (quimioterapia) en español, o también barbs (barbitúricos), Pap (prueba de Papanicolaou) y staph (estafilococo) en inglés $(8,9)$.

El término abreviatura no debe confundirse jamás con abreviación; este último se refiere al acortamiento de una o varias palabras mediante cualquiera de los métodos de abreviación, mientras que el primero se refiere únicamente a uno de ellos. Por ello, abreviar correctamente es tan importante como emplear adecuadamente cualquier vocablo. Otro rasgo muy importante y que ayuda mucho a distinguir las abreviaturas de las demás formas de abreviación es que la lectura de una abreviatura debe restablecer todas las letras eliminadas en su escritura; es decir, debe leerse la palabra completa que la abreviatura representa, a diferencia de las siglas.

El fenómeno de abreviación en la lengua suele ser muy común, tanto en la lengua oral como en la escrita y en las lenguas de especialidad; siendo su origen el principio de la economía del lenguaje y la ley del mínimo esfuerzo por parte de los usuarios de la lengua. Las siglas constituyen uno de los métodos de enriquecimiento del léxico más característicos de nuestro siglo. Las abreviaturas son utilizadas con elevada frecuencia en el discurso médico especializado, tanto por estudiantes de pre y posgrado en medicina como por los médicos generales, los especialistas, los supra-especialistas y otros profesionales de la salud. Suelen utilizarse para economizar espacio y tiempo y para evitar el empleo de nombres de enfermedades o procesos graves, incurables o vergonzosos. Muchas de ellas no están consensuadas por la comunidad y son inventadas, por lo que resultan difíciles de entender y entorpecen el flujo de la comunicación entre los diversos niveles asistenciales y la correcta transmisión del conocimiento, es por ello que el lenguaje médico muestra una serie de variedades en su uso $(7,8,9)$.

La estimación que hacen hoy en día muchos filólogos es que hay más de medio millón en términos médicos, debido a que estamos en la presencia de un lenguaje que se acrecenta al mismo tiempo que progresa la ciencia médica. El problema en este crecimiento de la terminología médica es la incorporación constante de neologismos con anglicismos y de la falta de normalización, lo que fomenta una complejidad léxica que provoca incorrecciones y dudas frecuentes en los propios médicos y en el ciudadano común.

Los problemas que se desatan con el uso de abreviaciones son muy variados y complejos; desde la interpretación variable en función del contexto o la lengua, en la que está expresada (polisemia), asimismo la falta de una norma uniforme en su elaboración, y por último, el ahorrar tiempo y espacio, no siempre se ha conseguido con el uso de las abreviaciones, sino que todo lo contrario; lejos de enriquecer el lenguaje representa una intrincada tarea de descifrado cuando la abreviatura se convierte en algo ininteligible (8).

El uso de abreviaciones constituye uno de los principales atropellos que se producen en el lenguaje médico y es el propio profesional quien sufre las consecuencias. Prácticamente en todos los documentos médicos asistenciales se clasifican en abreviaturas, siglas y acrónimos. Las siglas corresponden a la representación de una palabra o conjunto de palabras mediante la 
letra inicial de cada una de ellas, por ejemplo, VIH (virus de inmunodeficiencia humana). Los acrónimos no son siglas, sino palabras formadas al descomponerse otras, sean o no iniciales como DMN (diabetes mellitus neonatal) $(8,9)$. En torno a lo dicho, se elaboró la confección de una lista de abreviaciones, las más conocidas y utilizadas en el lenguaje médico, como se expone en la tabla 2.

\section{Razones de su existencia en el lenguaje médicoo}

El lenguaje médico, como todo lenguaje científico, no persigue fines estéticos, creativos, lúdicos ni recreativos como sería el caso del lenguaje literario, sino fines informativos, didácticos y comunicativos (1-9).

Tabla 2. Abreviaturas más conocidas en el lenguaje médico y su significado.

\begin{tabular}{|c|c|c|c|}
\hline ABREVIATURA & SIGNIFICADO & ABREVIATURA & SIGNIFICADO \\
\hline a.Ce & Antes de la cena & Bx & Biopsia \\
\hline a. Co & Antes de la comida & Ca & Carcinoma \\
\hline a. De & Antes del desayuno & ECG & Electrocardiograma \\
\hline AAN & Anticuerpos antinucleares & Eco & Ecografía \\
\hline ADB & Abdomen y abducción & EEG & Electroencefalograma \\
\hline ABT & Antibiótico & Epi & Epidural \\
\hline Ac & Anticuerpo & Espir & Espirometría \\
\hline $\mathrm{AC}$ & Auscultación cardiaca normal & Expec & Expectoración \\
\hline Adenop & Adenopatías & $\mathrm{HCl}$ & Historia clínica \\
\hline HAD & $\begin{array}{l}\text { Hormona antidiurética o } \\
\text { vasopresina }\end{array}$ & Hct & Hematocrito \\
\hline ADN & Ácido desoxirribonucleico & Dr & Doctor \\
\hline Anat & Anatomía & Dra & Doctora \\
\hline Anat. Pat & Anatomía patológica & Dto & Desprendimiento \\
\hline Antec & Antecedentes & Cardio & Cardiología \\
\hline Ao & Aorta & Cef & Cefálica \\
\hline $\mathrm{AO}_{2}$ & Contenido arterial de oxígeno & Cta & Consulta \\
\hline Aprox & Aproximadamente & Cta & Cucharadita \\
\hline Ausc & Auscultación & $\mathrm{Gg}$ & Grageas \\
\hline$A x$ & Axila & Cap & Cápsula \\
\hline BAL & Broncoalveolar & VO & Vía oral \\
\hline Bil & Bilirrubina & Susp & Suspensión \\
\hline Bilat & Bilateral & Tab & Tableta \\
\hline Bip & Bipedestación & TBC & Tuberculosis \\
\hline B1 & Blando & VN & Valor Normal \\
\hline Bleo & Bleomicina & WPW & $\begin{array}{l}\text { Síndrome de Wolff } \\
\text { Parkinson White }\end{array}$ \\
\hline Quimio & Quimioterapia & & \\
\hline
\end{tabular}


médicoEs por ello que uno de los rasgos más característicos en el lenguaje de las ciencias de la salud, por un lado, es el uso de epónimos o términos construidos sobre nombres propios. Se ha pensado que el uso de epónimos en las ciencias médicas contribuye a su universalización, mientras que las abreviaturas, siglas y acrónimos en las lenguas de las distintas especialidades como en la medicina, establecen una de sus principales características léxico-semánticas. Pese a todo esto, los epónimos y las abreviaciones han hecho que, por tradición y uso, se haya impuesto su empleo en el lenguaje médico y en el quehacer diario de la práctica médica (4).

El lenguaje médico está lleno de epónimos, bien sea con un nombre propio con el cual se designa una enfermedad, un síndrome, un signo, una maniobra, una técnica quirúrgica o un dispositivo. Estos epónimos se aprenden desde la escuela de medicina y son utilizados por los médicos y otros profesionales, trasmitiéndose su uso de generación en generación (10).

Son muchos los argumentos o razones esgrimidas que justifican la existencia de os epónimos en el lenguaje médico actual: 1. Contribuyen a facilitar la comunicación entre pares. 2. Han sido la forma más fácil y sencilla de nombrar los nuevos descubrimientos; la mayoría de las veces la descripción de los mismos, precede al entendimiento completo de los términos médicos. 3. Constituyen un justo homenaje a la perspicacia clínica y capacidad de observación de sus inventores, descubridores o investigadores. 4. Cuando este se percibe bien en su contenido, su uso revela notoriamente un ahorro en la comunicación médica; de no emplearlos, en cada caso habría que hacer una exposición o descripción relativamente amplia de la enfermedad o el signo de que se trata. 5. Aportan aspectos humanísticos sobre la investigación científica, al tiempo que proporcionan una perspectiva histórica de los diferentes dominios de las ciencias. 6 . Resulta una forma económica desde el punto de vista lingüístico para la comunicación entre especialistas, ya que permiten simplificar denominaciones excesivamente largas. 7. Nos topamos con una realidad muy generalizada y extendida, la cual ha coexistido durante gran parte de la historia de la humanidad. 8. Su uso no se limita exclusivamente al área de las ciencias médicas, sino que estos también están presentes en todos los campos del conocimiento, de la vida y se asignan con frecuencia a todo tipo de realidades; de hecho, es un fenómeno tan frecuente, que algunos epónimos ya no llevan mayúscula, puesto que se han incorporado completamente al lenguaje del día a día. 9. Sirven en ocasiones para cubrir un vacío existente en vez de requerir a una creación. 10. Son simples y concisos por lo que se evitan escribir o recitar grandes definiciones, no exentas de generar tergiversación de los términos a lo que se refieren $(9,10,11)$.

Los epónimos surgen como respuesta necesaria a la necesidad de modas y nuevas denominaciones, a modo de neologismos y los medios de comunicación y difusión son los principales propagadores de ellos, de su incorporación en el idioma y de los cambios en su estructura y significado; también se encuentran íntimamente vinculados al proceso de transmisión del conocimiento.

En cambio, el uso de abreviaciones se ha introducido en el lenguaje médico-científico con el objetivo inicial de ganar tiempo y ahorrar espacio y, si se emplean con discreción, simplifican las expresiones complejas $(7,8)$. Las abreviaciones, para muchos son una necesidad de "economía lingüística". Cada vez tiene mayor auge en las notas de los profesionales de la medicina, que se ha convertido en un lenguaje en clave.

Actualmente se ha hecho muy habitual el uso de las abreviaturas, así como de las siglas y acrónimos, siendo utilizados en cualquier faceta de la vida cotidiana. Pero no cabe la menor duda de que estas son muy dinámicas, ya que continuamente se crean nuevas abreviaciones, 
dejan de utilizarse otras, cambian de significado o se añaden nuevos significados a las ya existentes. La abreviatura es una codificación de la escritura donde su rasgo esencial descifrado, es que estas solo funcionan en el plano de la escritura, pues a la hora de su lectura se reconstruye en su totalidad lo abreviado. En la actualidad existen más de 42.000 palabras con sus abreviaturas (9), y en la documentación científica actual se crean continuamente abreviaturas, siglas y acrónimos, muchas de ellas reconocidas internacionalmente como ISBN (International Standard Book Number) e ISBD (International Standard Bibliographic Description) (12).

Los fenómenos de la abreviación de la lengua suelen ser muy comunes, tanto en el lenguaje común, escrito y oral, sobre todo en el lenguaje del discurso especializado médico (historia clínica), tanto por estudiantes de pre y posgrado en medicina como por médicos generales, médicos especializados, médicos supra-especializados y otros profesionales de la salud, siendo su origen el principio de la economía del lenguaje y la ley del mínimo esfuerzo por parte de los usuarios. Las siglas son un importante aliado en los textos médicos y su misión es evitar tediosas repeticiones de largas denominaciones (13).

\section{Principales problemas que plantea su uso en el lenguaje médico actual}

Recordemos que el lenguaje médico, como todo lenguaje científico, suele echar mano de términos muy precisos en busca siempre del ideal inalcanzable de la correspondencia biunívoca entre significantes y significados, de tal modo que cada concepto, cada idea, cada entidad tenga una sola palabra para designarlo, y cada palabra, cada término, designe un único concepto (14). Es evidente el riesgo de confusión cuando se utilizan términos imprecisos que autor y lector pueden interpretar de forma distinta. Es muy recomendable, desde luego, evitar en el lenguaje científico el uso de términos que admitan más de una interpretación como es el uso de los epónimos que ya hoy en día no están justificados, y por tanto, se hace necesario sustituirlos términos más precisos; se debe realizar un esfuerzo consciente para evitar su uso y con ello, erradicarlo del lenguaje médico y en su lugar, se estima que sería más pertinente utilizar términos construidos de acuerdo con la nomenclatura médica normalizada (14).

Los nombres epónimos difieren de un país a otro, por lo puede originar la modificación en su escritura al momento de su traducción, lo que ha dado motivo para confusiones que hacen muchas veces difícil el poder identificar su verdadero significado y que no dejan de crear en ocasiones un verdadero caos, que genera cierta dificultad a la hora de comunicarse entre el personal médico de las diferentes partes del mundo. Algunos nombres epónimos utilizados son anglosajones, lo que produce en ocasiones confusión, pues el primer problema que plantean es escribirlo correctamente y, el segundo es que, en ocasiones, se trasforman en adjetivos. Sin embargo, sustituir un epónimo por un sinónimo no está exento de problemas, pues puede producir una confusión mayor. Los nombres epónimos no tienen precisión científica (15).

Los epónimos dan pie a la confusión, pues a veces hay más de un epónimo para designar un solo concepto (sinonimia), lo que trae consigo un gran inconveniente tanto para la exactitud del lenguaje médico como para la comprensión histórica del mismo. El uso de los epónimos no deja de ser una estigmatización o despersonalización. Parecería que la persona ya no es un enfermo que tiene una enfermedad, sino un síndrome $(15,16)$.

Mientras que los problemas que conlleva el uso de abreviaciones son múltiples: interpretación variable en función del contexto o la lengua en la que está expresada (polisemia), falta de un criterio uniforme en su confección, complejidad al lexicalizarse (por ejemplo 'sidoso' o 'sídico', cuando se transforma en adjetivo el léxico 'sida', que inicialmente era el acrónimo del síndrome de inmunodeficiencia adquirida). 
Su uso tenía como objetivo inicial ahorrar tiempo y espacio y ganar en claridad, esto no siempre se ha conseguido, al contrario; lejos de enriquecer el lenguaje representa una ardua tarea de descifrado cuando la abreviatura se convierte en algo ininteligible (7). Otro inconveniente proviene del hecho de que algunas abreviaciones tienen varios significados diferentes (abreviaciones polisémicas), por lo que una misma abreviación puede interpretarse de diferentes maneras por diferentes médicos y en cada caso habrá que elegir el significado que pueda corresponder al texto en cuestión, con el consiguiente peligro de producir falsas interpretaciones.

Desde el punto de vista lingüístico, las abreviaturas suplantan en su función a los elementos naturales de la lengua, no se someten a reglas morfológicas, carecen de acepción y no pueden ser consideradas como símbolos. Las abreviaturas son un instrumento impreciso y peligroso porque contradicen las normas básicas del lenguaje científico (que postula el sentido unívoco y denotativo de los términos), no pertenecen a un sistema que esté al amparo de convenciones establecidas, sino que es coyuntural y depende del capricho del creador o editor $(13,14)$. Muchas veces es difícil recordar el significado original de las palabras de las que provienen, ya que son términos artificiales que carecen de derivación etimológica.

Las siglas son una necesidad en la "economía lingüística", de uso cada vez mayor en el lenguaje escrito de los profesionales en salud, se han convertido en un léxico de clave y su uso indiscriminado, entraña peligros de ininteligibilidad, siendo elemento de separación o incomprensión en la comunicación.

La mayor parte de las siglas no se han adoptado por acuerdo de la comunidad internacional ni están amparadas por los comités de normalización, sino que son inventadas. En algunas ocasiones la sigla es tan compleja que puede resultar incluso más difícil de recordar que la propia palabra o frase a la que representa, este abuso de las siglas afecta de manera fundamental a la comunicación interprofesional en la práctica médica, especialmente a los informes médicos. Es bastante frecuente recibir informes de médicos de otra especialidad que, debido a un exceso de siglas, resultan prácticamente ininteligibles de signos difícilmente descifrables para el destinatario, haciendo inútil el trabajo realizado al escribirlo. Esto se debe a que uno de los problemas de esta práctica, es que algunas siglas tienen varios significados (polisemia), similitudes (sinonimia) o ambigüedades (anfibología) y otras corresponden a anglicismos (extranjerismos), dando lugar a que una misma sigla sea interpretada de diferente forma por varios profesionales de la salud, con el resultado final de producir falsas interpretaciones, y conllevar al error médico. Es posible que con su uso, sobre todo en las siglas particulares o inventadas, algunos profesionales de la salud traten de minimizar el impacto que pueda ocasionar la palabra o frase completa, como en enfermedades socialmente dolorosas como el cáncer ( $\mathrm{Ca})$, incurables como el síndrome de inmunodeficiencia adquirida (sida) o vergonzosas como la tuberculosis (TBC). Como consecuencia, en los últimos años, en el ámbito médico, se ha visto con frecuencia la utilización de siglas que en la mayoría de los casos no están homologadas nacional ni internacionalmente.

Finalmente, es por esto que en el lenguaje científico, el abuso de abreviaturas, siglas y acrónimos que no han sido internacionalmente aceptados por los comités de normalización convierte al lenguaje en un instrumento impreciso, con graves problemas para su comprensión, ya que en ocasiones se establecen por simple economía lingüística del creador.

\section{CONCLUSIÓN}

En el momento actual no son pocas las voces que opinan en torno a la pertinencia que tiene el empleo de epónimos, abreviaciones, siglas y acrónimos, ya que para estos son instrumentos 
imprecisos y peligrosos, sujetos al capricho del creador y carentes de equivalencia internacional, lo que puede llevar a confundir la realidad que tratan de describir, ya que una misma abreviación puede tener diferentes significados. Además, las abreviaciones evolucionan, aparecen otras nuevas que las sustituyen, pierden o cambian de significado, se utilizan en nuevas situaciones en las que es difícil reconocerlas o caen en desuso. He aquí que muchos apoyan su erradicación total o normatización por los comités de normalización, dentro del lenguaje de las ciencias médicas. Sus argumentos no son triviales, puesto que los problemas que plantean los epónimos, abreviaciones, siglas y acrónimos, son numerosos, tal y como hemos puesto de manifiesto a lo largo de esta revisión.

\section{REFERENCIAS}

1. Jiménez, GI. La sinonimia y la polisemia en la terminología anatómica: términos de ubicación y de relación de estructuras anatómicas. Entre culturas. 2009; 1:579-97.

2. Gutiérrez Rodilla. Lo literario como fuente de inspiración para el lenguaje médico, Panacea. 2003; 4 (11): 61-67.

3. Sempere, J. Problemas terminológicos en medicina: ¿alguna novedad? Papeles Médicos 2001; 10(3):136-143.

4. López Piñero JM, Terrada ML. Introducción a la terminología médica. Barcelona: Salvat; 1990.

5. Esteban-Arrea, C. La eponimia en el lenguaje científico. Razones de su existencia y principales problemas que plantea. 2012. Trabajo de grado. Universidad de Salamanca, Facultad de Traducción y Documentación. p: 8-55.

6. González-López, E. ¿Hay que seguir utilizando algunos epónimos médicos?, Medicina Clínica. 2010,134 (15): 703-704.

7. Aleixandre-Benavent, A., Alonso-Arroyo, M. González-Muñoz, J. González de Dios, x. Comunicación científica (XXIII). Lenguaje médico (1): Usos y abusos de las abreviaturas y siglas en el lenguaje médico y en pediatría. Acta Pediatr Esp. 2015; 73(5): 134-140

8. Rodríguez-Gama, Á, Donado-Moré, AF, Salcedo-Quinche, MP. (2014). Reflexiones en torno a los epónimos en medicina: presente, pasado y futuro. Revista de la Facultad de Medicina, 62(2): 305-317.

9. Aleixandre-Benavent, A., Porcel A, Agulló A, Marset S. Vicios del lenguaje médico (I). Extranjerismos y acrónimos. Atención Primaria 1995; 15 (2): 113-118.

10. Pulido J, Matteson E. Eponyms: What's in a Name? Retina. 2010; 30:1559-60.

11. Goic A. Sobre el uso de epónimos en medicina. Rev Med Chil. 2009; 137:1509.

12. Aleixandre-Benavent R. et al. Uso y abuso de abreviaturas y siglas entre atención primaria, especializada y hospitalaria. Papeles médicos 2006; VOL. 15 (2): 29-33.

13. Puente $\mathrm{CJ}$. Siglas y abreviaturas del inglés que son de utilidad en cirugía pediátrica, pediatría y neonatología. Panacea. 2003; 4: 131-135.

14. Aleixandre R, Amador A. Vicios del lenguaje médico y defectos de estilo en la escritura científicomédica (II). Piel. 2003; 18: 11-6.

15. Ezpeleta E. Virtudes e inconvenientes de los epónimos médicos. Ed. Esmon pharma. Barcelona, 2004: 64.

16. Aleixandre-Benavent, R.; Amador Iscla, A. Problemas del lenguaje médico actual. (II) Abreviaciones y epónimos. Papeles Médicos, 2001; 10(4):170-176. 